\title{
PHENOMENOLOGICAL THEORY OF ANISOTROPIC ISOTHERMAL GALVANOMAGNETIC EFFECTS*
}

\author{
L. P. KAO and E. KATZ \\ Physics Department, The University of Michigan, Ann Arbor, Michigan
}

(Received 13 December 1957)

\begin{abstract}
The isothermal galvanomagnetic tensor components associated with the Hall effect and the magnetoresistance effect are analyzed for arbitrary orientation of the crystal axes in the sample, arbitrary orientation of the magnetic field $B$, and arbitrary crystal symmetry. The conductivity components in suitable co-ordinates are expanded in powers of the components of $B$. The coefficients are the galvanomagnetic material constants, called "brackets". By means of ONSAGER's relations, it is shown that the magnetoresistance effect is always even in $B$, whereas the Hall effect is odd in $B$ only with special geometry. Further, the Hall effect is even or zero for some geometric and crystallographic conditions. The effects of the crystal symmetry on the brackets are covered by a theorem. The resultant dependencies between the brackets are tabulated completely for all powers of $B$ for the crystal classes other than trigonal and hexagonal. For the latter, the bracket relations are given up to the sixth power of $B$. Formulae for the number of independent brackets up to any power of $B$ are given for all crystal symmetries. The significance of the results obtained is pointed out.
\end{abstract}

\section{INTRODUCTION AND MOTIVATION}

IN an isothermal $\ddagger$ single crystal, placed in a homogeneous magnetic field $B$, a constant current density $J$ is maintained by means of a suitable electric field $F$ (see Fig. 1). Evidently

$$
F=F(J, B) \text {. }
$$

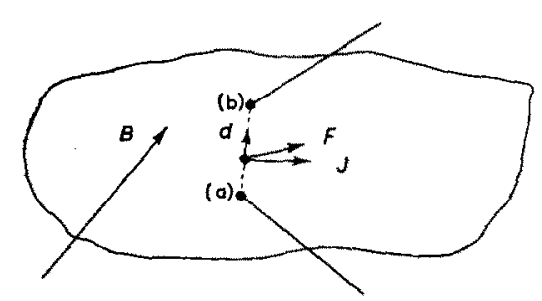

F1G. 1. Orientation of vectors $J, F, B$ and potential probes $a b$ in an anisotropic single crystal.

The dependence of $F$ on $B$ represents the galvano-

* This work was supported by the U.S. Signal Corps Engineering Laboratories under a contract with The University of Michigan.

+ This work represents, in effect, part of the doctoral thesis of L. P. KAJ (1956).

$\$$ Isothermal conditions are assumed throughout this paper without further explicit statement. magnetic effect. $\S$ The component $F^{d}$ of $F$ along an arbitrary direction $d$ can be measured by means of potential probes $a b$. If $d$ is parallel to $J$, the resulting dependence of $F_{y}(J, B)$ is called the magnetoresistance effect; if $d$ is normal to $J$, then $F_{\perp}(J, B)$ is called a Hall effect.|| Both are special cases of the galvanomagnetic (g.m.) effect.

The early literature is summarized by CAMPBELL (1) and by MeIsSNer. (2) Both effects are markedly dependent on the crystal structure and

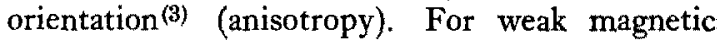
fields the Hall effect is known to be proportional to $B$, and the magnetoresistance effect is proportional to $B^{2}$. For stronger fields, and especially at lower temperatures, the dependences are much more complicated. ${ }^{(4-11)}$ In 1905 VoIGT $^{(12)}$ laid the foundation for an appropriate description of the anisotropy of the g.m. effects. Further contributions to the phenomenological theory were made by Konler, (13) Casimir, (4) SeItz, (14) JuRETSChKe, (15).

$\S$ Throughout this paper we restrict ourselves to nonferromagnetic substances.

II We shall adhere to this definition, though some experimenters prefer to define the Hall effect as measured with $d$ along an equipotential when $B=0$. 
and others, but a general treatment of the anisotropy was not available up to the present.

The objective of the present paper* is to generalize the phenomenological theory of the anisotropic g.m. effects, so as to include all magnitudes of $J$ and $B$, oriented arbitrarily with respect to the axes of a crystal belonging to any symmetry group. The results may assist experimenters in deciding how many and what types of measurements are required and how data can best be compared; theoreticians may find a framework with which model theories must comply.

In Section 2 some general definitions and relations are established. In Section 3 a general method is developed to take into account the effects of crystal symmetry. In Section 4 formulae are given for the number of independent g.m. constants for the various crystal classes. In Section 5 the significance of the results is discussed. We have purposely avoided in this paper to introduce any model of the mechanism by means of which these complex phenomena may take place.

\section{DEFINITIONS AND GENERAL RELATIONS}

\section{(a) Parity relations}

The results of g.m. measurements are best described in terms of "laboratory co-ordinates" $x^{\alpha}(\alpha=1,2,3)$ with $x^{1}$ along the current density $J, x^{2}$ in the plane of $J$ and $d$, and $x^{3}$ accordingly. In the case of magnetoresistance, $d$ lies along $J$, allowing one degree of freedom for $x^{2}$ and $x^{3}$ in the plane normal to $x^{1}$. Vector and tensor components with respect to the laboratory co-ordinates will carry Greek superscripts. No confusion between superscripts and exponents should arisc in practice. The definition of the laboratory system implies

$$
J^{2}=J^{3}-0 .
$$

We assume Ohm's law. In laboratory co-ordinates:

$$
F^{\alpha}=\rho^{\alpha 1}(B) J^{1} \text {. }
$$

The g.m. resistivity tensor components $\rho^{\alpha 1}$ depend on $B$. We assume also Onsager's relations. In

* The essential ideas of the present paper were first given by the authors at the Baltimore meeting of the American Physical Society in $1955^{(16)}$ and by E. KATz in a contract report, with the U.S. Signal Corps Engineering Laboratories, of November, 1954, entitled: "Magnetically Sensitive Electrical Resistor Material." laboratory co-ordinates:

$$
\rho^{\alpha \beta}(B)=\rho^{\beta \alpha}(-B) .
$$

Taking $\alpha=\beta=1$ in equations (3) and (4), one sees that the magnetoresistance is an even function of $B$. In the literature ${ }^{(17)}$ there has been some controversy about the evenness of $p^{11}$. 'The above approach shows that under the very broad assumptions stated, $\rho^{11}$ must be an even function of $B$ without exception. Taking $\alpha \neq 1$ in equations (3) and (4), one sees that the Hall effect is in general neither an odd nor an even function of $B$, contrary to a suggestion by CASIMIR. ${ }^{(4)}$ However, in a number of special configurations the crystal symmetry may impose an even or odd parity on the Hall effect. The complete list of such configurations is as follows. Consider the crystallographic point group, obtained from that of the crystal by augmenting it with an inversion center. The physical significance of this augmented group is explained in Section 3. Then one can easily prove with respect to the rotation axes of this augmented group:

(1) The Hall effect is odd if either

(a) $B$ lies along a rotation axis of order higher than 2 and either $J$ or $d$ is normal to $B$, or if

(b) $B$ is normal to an axis of even order and either $J$ or $d$ is along that axis.

(2) The Hall effect is even if either

(a) $B$ lies along any rotation axis and is coplanar with $J$ and $d$, or if

(b) $B, J$, and $\alpha$ are normal to the same axis of even order.

(3) The Hall effect vanishes if $B$ and either $J$ or $d$ lie along the same arbitrary rotation axis.

The "new" galvanomagnetic effect reported by GoldBERG and DAvis ${ }^{(18)}+$ illustrates cases $(2 b)$ and (3).

\section{(b) Symmetry co-ordinates}

For the purpose of deriving the effects of crystal symmetry, it is convenient to refer to "symmetry co-ordinates" $k_{i}(i=1,2,3)$. These are adapted to the crystallographic point group of the crystal, augmented again by an inversion center, as follows:

$\uparrow$ The slight discrepancy between the axis direction and the direction of zero Hall effect in their Fig. 1 must be due to an experimental error of imperfect alignment. 
$k_{3}$ is taken along the rotation axis of highest order, except for $T_{h}$ where it is taken along a twofold axis. Then $k_{1}$ is taken along a rotation axis normal to $k_{3}$ if there be one, and $k_{2}$ accordingly. Vector or tensor components with respect to the symmetry co-ordinates will carry Latin subscripts. The components $\sigma_{i j}(B)$ of the conductivity tensor and $\rho_{i j}(B)$ of the resistivity tensor are functions characteristic of the material at any given temperature and independent of the geometry of galvanomagnetic measurements. The crystal symmetry will place restrictions on them.

\section{(c) Power series expansion of $\sigma_{i j}(B)$}

Most g.m. measurements suggest ${ }^{(19)}$ that $\sigma_{i j}(B)$ can be expanded as a series in powers* of the components $B_{1}, B_{2}, B_{3}$. Thus:

$$
\begin{aligned}
& \sigma_{i j}(B)=\sum_{n=0}^{\infty} \sum_{m=0}^{n} \sum_{p=0}^{m}[m-p, p, n-m]_{i j} \times \\
& \times B_{1}^{m-p} B_{2}^{p} B_{3}^{n-m} \\
& =\sum_{n=0}^{\infty} B^{n} \sum_{m=0}^{n} \sum_{p=0}^{m}[m-p, p, n-m]_{i j} \times \\
& \times \gamma_{1}^{m-p} p_{\gamma_{2}} p_{\gamma_{3}}{ }^{n-m} .
\end{aligned}
$$

Here $\gamma_{i}$ are the direction cosines of $B$ with respect to the symmetry co-ordinates. The coefficients, the "brackets" symbols, are independent of $B$. They are the true phenomenological g.m. constants for each material at a given temperature. They are sums of components of tensors $\uparrow$ of rank $2 n+2$, since $B$ is an antisymmetric tensor of rank two.

\footnotetext{
* Such a power series expansion may have a limited domain of convergence, for example, if saturation is approached according to $B^{2} /\left(1+C B^{2}\right)$. Also, experimental ${ }^{(20)} \mathrm{g} \cdot \mathrm{m}$. effects at low temperatures often contain oscillatory terms of the form $B \sin B_{0} / B$, which do not possess a derivative with respect to $B$ at $B=0$, and hence cannot be expanded in powers of $B$ (de Haas-van Alphen effect). Consequently, the development presented here applies only within the radius of convergence of the power series (7), and after that part of the g.m. effects which is due to oscillatory terms has been subtracted out, if present.

$f$ In order to set the tensor character in evidence, the brackets will sometimes be denoted by $\left[(23)^{m-p},(31)^{p}\right.$, $\left.(12)^{n-m}\right]_{i j}$. The quantities (23), (31), (12) will be referred to as the pairs of inner indices and $i j$ as the outer indices.
}

Onsager's relations imply

$$
[m-p, p, n-m]_{i j}=(-)^{n}[m-p, p, n-m]_{f_{i}}
$$

and

$$
[m-p, p, \omega-m]_{i i}=0
$$

where $\omega$ is any odd number. Thus only six pairs of $i j$ values will be used: $11,22,33,23,31,12$. The remaining three pairs are expressed in the former by equation (8).

The effects of crystal symmetry on the measured functions $\rho^{a 1}(B)$ are obtained in two steps which will be discussed in this order:

(a) The anisotropy effects on the brackets (coeffcients of the expansion) of $\sigma_{i j}(B) \ddagger$ (see Sections 3 and 4). 5).

(b) The dependence of $\rho^{\alpha 1}(B)$ on $\sigma_{i j}(B)$ (Section

\section{THE EFFECTS OF CRYSTAL SYMMETRY}

In this section a method will be developed and applied to take into account the effects of crystal symmetry on the brackets under a wide variety of circumstances.

\section{(a) Only eleven point groups need analysis}

Material constants which are components of even rank tensors must be invariant under the operations of the point group of the crystal considered and transform identically into themselves under inversion. Consequently the brackets must be invariant under the operations of the augmented point group of the crystal plus an inversion center. Thus it suffices to analyze the eleven point groups which possess such a center. They can all be generated by at most two rotations in addition to the inversion center. The first generating rotation will always be taken along $k_{3}$ and where a second one is required, along $k_{1}$ except for the cubic groups, where it will be taken along the [111] direction.

Under a general rotation each bracket is transformed into a linear combination of other brackets. If the rotation be a covering operation, and thus

\# The measurement of $\rho^{\alpha .1}$ would scem to suggest a power series expansion of $\rho_{i j}(B)$ instead of $\alpha_{i j}(B)$. The latter is preferred because its coefficients admit of a simpler electron-theoretical interpretation. However, the symmetry properties of the $\rho$ and $\sigma$ brackets are exactly the same. 
requires invariance of the bracket, then certain relations must hold between the brackets.

Under inversion each bracket is transformed identically into itself. Hence, no relations between brackets can be derived from the requirement of invariance under inversion.

\section{(b) A theorem concerning the effect of an $N$-fold} rotation axis along $k_{3}$

The effect of an $N$-fold rotation axis is that certain linear equations must hold between the brackets. The following theorem makes it easy to produce and to survey all such equations.

For an $N$-fold rotation axis along $k_{3}$ the brackets satisfy the equations

$$
\sum_{i j} \sum_{p=0}^{m} g(m, p, w) \epsilon(\ddot{j}, z)[m-p, p, n-m]_{i j}=0
$$

provided that the quantity $h \equiv m+s-2(w+z)$ satisfies the inequality

$$
h \neq k N \quad(k=0, \pm 1, \pm 2, \ldots)
$$

i.e. $h$ is not a multiple of $N$.

Here the prime on the first summation signifies that of the six independent $i j$ combinations only those with the same number $s$ of ones plus twos are included. Each equation is specified by five integers $n, m, s, z, w$, where $n$ is the sum of the powers of $B_{1}$, $B_{2}$, and $B_{3}$ of which the brackets occurring in the equation are coefficients, $m$ is the sum of the powers of $B_{1}$ and $B_{2}, s$ is the number of ones plus twos in $i j$, and the integers $z$ and $w$ are arbitrary in their ranges $0 \leqslant z \leqslant s$ and $0 \leqslant w \leqslant m$. All brackets occurring in one equation have the same $n, m, s$, but differ in $p$, and in $i j$, insofar as is permitted by constant $s$. The parameters $z, w$ label the various equations with the same $n, m, s$, involving the same brackets with different coefficients. The real or imaginary coefficient $g(m, p, w)$ is defined in terms of binomial coefficients by

$$
g(m, p, w) \equiv i^{p} \sum_{q=0}(-)^{q}\left(\begin{array}{c}
m-p \\
q
\end{array}\right)\left(\begin{array}{c}
p \\
w-q
\end{array}\right) .
$$

The factor $\epsilon(i j, z)$ is given in Table 1 for all values for which it is defined. The proof of this basic theorem is given in the Appendix. It can be shown that the only solution for the complete set (9) for given $n, m, s$ is that all brackets involved vanish. Consequently the equations (9) with the conditions (10) represent a complete description of the sym-

\begin{tabular}{|c|c|c|c|c|c|c|c|c|}
\hline \multirow[b]{2}{*}{$s$} & \multirow[b]{2}{*}{$i j$} & \multirow[b]{2}{*}{$z \rightarrow$} & \multicolumn{3}{|c|}{$n=$ even } & \multicolumn{3}{|c|}{$n=$ odd } \\
\hline & & & 0 & 1 & 2 & 0 & 1 & 2 \\
\hline 0 & 33 & & 1 & - & - & - & - & - \\
\hline 1 & 31 & & 1 & 1 & - & 1 & -1 & - \\
\hline 1 & 23 & & $i$ & $-i$ & - & $-i$ & $-i$ & - \\
\hline 2 & 11 & & 1 & 1 & 1 & - & - & - \\
\hline 2 & 12 & & $2 i$ & 0 & $-2 i$ & 0 & $-2 i$ & 0 \\
\hline 2 & 22 & & -1 & 1 & -1 & - & - & - \\
\hline
\end{tabular}
metry properties of the brackets.

Table 1. The values of $\epsilon(i j, z)$

For example, the equation for $n=2, m=2$, $s=2, z=1$, and $w=0$ is $[200]_{11}+[200]_{22}+$ $+i[110]_{11}+i[110]_{22}-[020]_{11}-[020]_{22}=0$. The equation is valid provided that $h=2 \neq k N$, i.e. $k_{3}$ may be a $3-, 4-$, or 6 -fold axis.

By suitably combining the equations (9) with the same $n, m, s$ and different $w, z$, the brackets with these $n, m, s$ values can be solved in terms of a few of them which are independent (see Section 4). These solutions are tabulated below. Before describing the results it is expedient to observe certain general properties of the equations (9), which have guided the form in which the tables could be constructed.

\section{(c) Some consequences of the theorem}

In formulating the fundamental theorem, a rotation axis was taken along $k_{3}$. It is simple to apply the theorem to a rotation axis along $k_{1}$ or $k_{2}$ by permutation of both inner and outer indices. The effect of a threefold axis along the [111] direction can be taken into account by requiring invariance for the brackets under cyclic permutation of the indices 1, 2, 3 both in and outside any bracket. Thus the effect of symmetry for the eleven point groups is completely described by the theorem with these generalizations. However, in a number of cases the application of the theorem is greatly simplified by means of some corollaries, which are stated below. Their proofs are straightforward and are left to the reader. 


\section{Corollaries}

(I) For $N=$ even about $k_{3}$, brackets with the index 3 occurring an odd number of times (inside plus outside, the inside being written in the notation of pairs of indices) vanish.

In preparation of Corollary (II) let two brackets be called "adjoint" with respect to $k_{3}$, if they can be obtained from one another by interchanging the indices 1 and $2, *$ both inside and outside, and writing the resulting pairs of indices in the conventional order. For example, the brackets

$$
[m-p, p, n-m]_{23} \text { and }[p, m-p, n-m]_{31}
$$

are adjoint.

(II) For $N=4$ about $k_{3}$, non-vanishing adjoint brackets are either equal or opposite. They are equal if the number of occurrences of the index 2 is even, opposite if this number is odd. The same is true for the index 1 .

(III) If in an equation of the type (9) each bracket $[m-p, p, n-m]_{i j}$ is replaced by $[m-p$, $\left.p, n^{\prime}-m\right]_{i j}$, where $n^{\prime}$ has the same parity as $n$, the resulting equation also belongs to the set (9) and has the same $h$.

(IV) Two equations of the type (9) with equal $n, m$, s having parameter values $w, z$ and $w^{\prime}=m-v$, $z^{\prime}=-s-z$, hence $h^{\prime}=-h$, are conjugate complex.

(V) If in an equation of the type (9) each bracket is replaced by its adjoint, the resulting equation also belongs to the set (9) and has the same $|h|$.

The corollaries (I-V) are useful in tabulating the relations between the brackets for the various crystal classes. The corollaries (VI-IX) are useful as checking relations.

(VI) Any relation between brackets with $s=1$ is invariant for interchange of $i j=23$ and 31 , followed by reversal of the sign of the coefficients of all terms with $i j=31$.

(VII) Any relation between brackets with $s=2$ is invariant for the interchange of $i j=11$ and 22 , followed by reversal of the sign of the coefficients of all terms with $i j=12$.

(VIII) Any relation between brackets for $s=1$, $n=$ even, is transformed to a valid relation for $n=$ odd with the same $m, p, s$ by changing the sign of all brackets with $i j=23$, and vice versa.

(IX) For $m=$ odd any relation between brackets

* Similarly we define adjoint with respect to $k_{1}$ (or $k_{2}$ ) by interchanging the indices 2 and 3 (or 3 and 1 ). is invariant for the substitution $p^{\prime}=m-p$ followed by reversal of the sign of the coefficients of all terms with $i j=31$ and 12.

\section{(d) The bracket relations for the eleven point groups}

(i) $S_{2}$. No relations other than (8) and (8a).

(ii) $C_{2 h}$ and $D_{2 h}$. The relations are given in Table 2. The effect of symmetry is manifest entirely in the vanishing of certain brackets; all nonvanishing brackets are independent. Thus, we have used three symbols as explained under the table to indicate the state of a bracket whose inner part is given by the second column and whose outer indices appear in the top row. The inner parts of brackets contain the symbols $e$ for an arbitrary even number and $\omega$ for an arbitrary odd one.

Table 2. Bracket relations for $C_{2 h}$ and $D_{2 h}$

\begin{tabular}{|c|c|c|c|c|c|c|c|}
\hline & $i j \rightarrow$ & 23 & 31 & 12 & 11 & 22 & 33 \\
\hline $\begin{array}{l}\mathbf{E} \\
\stackrel{\mathbf{y}}{\mathbf{u}} \\
\mathbf{I} \\
\mathbf{c}\end{array}$ & 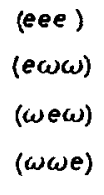 & $\begin{array}{l}\circ \\
+ \\
0 \\
0\end{array}$ & $\begin{array}{l}0 \\
+ \\
+ \\
0\end{array}$ & $\begin{array}{l}1 \\
0 \\
0 \\
+\end{array}$ & $\circ$ & $\begin{array}{l}+ \\
0 \\
0 \\
0\end{array}$ & $\begin{array}{l}+ \\
0\end{array}$ \\
\hline $\begin{array}{l}7 \\
8 \\
11 \\
\text { ? }\end{array}$ & $\begin{array}{l}(\omega \omega \omega) \\
(\omega e e) \\
(e \omega e) \\
(\text { ee } \omega)\end{array}$ & $\begin{array}{l}0 \\
+ \\
0 \\
0\end{array}$ & $\begin{array}{l}0 \\
0 \\
+ \\
0\end{array}$ & $\begin{array}{l}- \\
0 \\
0 \\
+\end{array}$ & & & \\
\hline
\end{tabular}

\footnotetext{
+ , bracket independent for $C_{2 \mathrm{~h}}$ and $D_{2 \mathrm{~A}}$.

$\rightarrow$, bracket independent for $C_{\mathrm{th}}$, zero for $D_{2 h}$. 0 , bracket zero for $C_{2 \mathrm{~A}}$ and $D_{2 \mathrm{k}}$.
}

Examples: $[203]_{23}$ is found to be zero as shown by $[e e \omega]_{23}$ in the eighth row, [204 $]_{12}$ is found to be independent for $C_{2 h}$ and zero for $D_{2 h}$ as shown by $[e e e]$ in the first row.

The outer indices $11,22,33$ cannot occur with $n=$ odd, according to equation (8a). Table 2 is complete for all $n$.

(iii) $C_{4 h}$ and $D_{4 h}$. The relations are given in Table 3. The effect of symmetry is manifest in two ways: either a bracket is zero or it is equal to plus or minus its adjoint (as defined in Corollary II of the theorem). Of each such pair of adjoint brackets, one bracket can be chosen as independent. 
Table 3. Bracket relations for $C_{4 h}$ and $D_{4 h}$

\begin{tabular}{|c|c|c|c|c|c|c|c|}
\hline & $i j \rightarrow$ & 23 & 31 & 12 & 11 & 22 & 33 \\
\hline \multirow{4}{*}{ 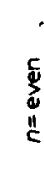 } & $(e e e)$ & o & 0 & $\dot{\theta}$ & & $-t$ & $t$ \\
\hline & $(e \omega \omega)$ & & & $\circ$ & & & 0 \\
\hline & (wew) & $\theta$ & $\psi$ & 0 & & o & 0 \\
\hline & (nowe) & o & 0 & + & & $-\theta$ & $\dot{\theta}$ \\
\hline \multirow{4}{*}{$\begin{array}{l}\text { गे } \\
\text { o } \\
\text { "I }\end{array}$} & $(\omega \omega \omega)$ & o & o & $\dot{\theta}$ & & & \\
\hline & (wee) & t & & 0 & & & \\
\hline & (ewe) & $\theta^{\prime}$ & ti & 0 & & & \\
\hline & $(e e \omega)$ & o & o & t & & & \\
\hline
\end{tabular}

$t$, bracket is one of an independent pair and equal to its adjoint for $C_{d h}$ and $D_{s h}$.

$\Theta$, bracket is one of an independent pair and equal to minus.its adjoint for $C_{4 h}$, zero for $B_{4 h}$.

$\dot{\theta}, \bullet$ and in addition zero if self-adjoint.

0 , zero for $C_{t h}$ and for $D_{t h}$.

Broken lines connect adjoint places.

In the first, fourth, fifth, and eighth or last row selfadjoint brackets may occur. A self-adjoint bracket may be forced to vanish if it must be minus its adjoint. Thus it turns out that four symbols are needed, whose meaning is explained under the Table 3, which gives the complete bracket relations for all $n$.

Table 4. Bracket relations for $T_{h}$ and $O_{h}$

\begin{tabular}{|c|c|c|c|c|c|c|c|}
\hline & $i j-$ & 23 & 31 & 12 & 11 & 22 & 33 \\
\hline \multirow{4}{*}{ 乌్ } & (eee) & 0 & $\circ$ & 0 & & & ++ \\
\hline & $(e \omega \omega)$ & 大 & 0 & o & 0 & o & o \\
\hline & $(\omega \in \omega)$ & $\circ$ & $+y$ & 0 & $\circ$ & $\circ$ & ○ \\
\hline & $(\omega \omega e)$ & $\circ$ & o & + & o & o & o \\
\hline \multirow{4}{*}{ 뭄 } & $(\omega \omega \omega)$ & $\circ$ & 。 & 0 & & & \\
\hline & (wee) & & o & $\circ$ & & & \\
\hline & $(e \omega e)$ & $\circ$ & $T$ & $\circ$ & & & \\
\hline & $(e e \omega)$ & & 0 & 4 & & & \\
\hline
\end{tabular}

+ , nonvanishing bracket for $T_{h}$ and $O_{h}$.

0 , zero bracket for $T_{h}$ and $O_{h}$.

In $T_{h}+$ is one of an independent cyclic set of three equal brackets. In $O_{h}+$ is one of an independent permuted set of three or six equal brackets (three, if brackets are pairwise self-adjoint). Broken lines indicate places of brackets of the same set.
Examples: $[202]_{12}=-[022]_{12}$ for $C_{4 h}$, zero for $D_{4 h}$ (first row). $[220]_{12}=$ self-adjoint and $-[220]_{12}$, hence zero for $C_{4 h}$ and $D_{4 h}$. $[202]_{11}=+[022]_{22}$; one of the pair is independent, both for $C_{4 h}$ and $D_{4 h}$.

(iv) $T_{h}$ and $O_{h}$. Table 4 for these groups is derived from Table 2 for $D_{2 h}$ and Table 3 for $D_{4 h}$ by requiring that brackets remain invariant under cyclic permutation of all indices. The six permutations of the indices 1, 2, 3 fall into two groups of three cyclic permutations. Brackets belonging to two such cyclic groups are pairwise adjoint with respect to $k_{1}, k_{2}$, and $k_{3}$. If adjoint brackets are to be equal, such as happens in $O_{h}$, or if brackets are pairwise self-adjoint, the two cyclic groups coincide. For example, for $T_{h}$ a cyclic group of three equal brackets, obtained by cyclic interchange of inner and outer indices from the first one, is:

$$
[202]_{11}=[220]_{22}=[022]_{33} \text {. }
$$

The other cyclic group of three equal brackets can be obtained from these by interchanging the indices 1 and 2, i.e. adjoining with respect to $k_{3}$ :

$$
[022]_{22}=[220]_{11}=[202]_{33} \text {. }
$$

For $T_{h}$ the two sets of three are not equal in general, but for $O_{h}$ all six are equal. On the other hand, the bracket $[220]_{33}$ is self-adjoint with respect to $k_{3}$; hence there is only one cyclic group of three brackets derived from it, and they are equal both for $T_{h}$ and $O_{h}$, namely:

$$
[220]_{33}=[022]_{11}=[202]_{22} \text {. }
$$

(v) $C_{3 i}, D_{3 i}, C_{6 h}, D_{6 h}$. No simple rules for the complete tabulation are available and the theorem plus corollaries will be used. In order to obtain the most compact form for the results, the following scheme has been adopted.

For any particular bracket we must first decide whether or not it is zero. For $C_{3 i}$ all zero brackets are listed in Table 5. The groups $D_{3 i}, C_{6 h}$, and $D_{6 h}$ have the same zeros as $C_{3 i}$ plus the additional zeros listed in Table 6. If a bracket does not vanish according to Tables 5 and 6 , then its relations to other brackets are shown in Table 7, up to $m=6$ inclusive. 
Table 5. Zero brackets for $C_{3 i} D_{3 i} C_{6 h} D_{6 h}$

\begin{tabular}{|c|c|c|c|c|c|c|c|}
\hline & $\ddot{i j \rightarrow}$ & 23 & 31 & 12 & 11 & 22 & 33 \\
\hline $\begin{array}{l}\mathbf{Q} \\
\mathbf{9} \\
\mathbf{0} \\
\text { II }\end{array}$ & 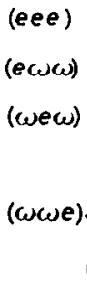 & $(\infty 0 e)$ & $\left(\infty e^{\prime}\right)$ & $(00 e)$ & & & $\begin{array}{l}(01 \omega) \\
(10 \omega) \\
(11 e) \\
(31 e) \\
(13 e)\end{array}$ \\
\hline $\begin{array}{l}\mathbf{7} \\
\mathbf{8} \\
\text { If } \\
\text { c }\end{array}$ & $\begin{array}{l}(\omega \omega \omega) \\
\text { (wee) } \\
\text { (ewe) } \\
\text { (eew) }\end{array}$ & 1006 & $(\infty, \omega)$ & $\begin{array}{l}(11 \omega) \\
(31 \omega) \\
(13 \omega) \\
(10 e) \\
(01 e)\end{array}$ & & & \\
\hline
\end{tabular}

Tablc 7 is arranged in thrce parts, according to $s=0,1$, or 2 . Each part consists of seven subtables for $m=0,1, \ldots 6$. The sub-tables, except the simplest ones, have the form of a core array of coefficients bordered by brackets. This arrangement represents a double-entry table, similar to

Table 6. Zero brackets for $\mathrm{D}_{3 \mathrm{i}} \mathrm{C}_{6 \mathrm{~h}} \mathrm{D}_{6 \mathrm{~h}}$

\begin{tabular}{|c|c|c|c|c|c|c|c|}
\hline & $i j \rightarrow$ & 23 & 31 & 12 & 11 & 22 & 33 \\
\hline \multirow{4}{*}{ 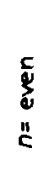 } & $(e e e)$ & * & $* \Delta$ & $\dot{\Delta}$ & & & \\
\hline & ( e $\omega \omega)$ & & $\Delta$ & $* \Delta$ & & * & $\dot{*}$ \\
\hline & (wew) & $\Delta$ & & * & $* \Delta$ & \# & $\Delta$ \\
\hline & $(\omega \omega e)$ & 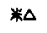 & * & & $\Delta$ & $\Delta$ & 8 \\
\hline \multirow{4}{*}{$\begin{array}{l}\text { 뭄 } \\
\text { c }\end{array}$} & $(\omega \omega \omega)$ & * & *A & $\dot{\Delta}$ & & & \\
\hline & (wee) & & $\Delta$ & *A & & & \\
\hline & $(e \omega e)$ & $\Delta$ & & * & & & \\
\hline & $(e e c u)$ & *A & $\dot{*}$ & & & & \\
\hline
\end{tabular}

$\Delta$, zero bracket for $D_{\mathbf{a}}$ and for $D_{\mathrm{ak}}$.

* , zero bracket for $C_{* h}$ and for $D_{\mathrm{A}}$.

$\because$ Is a reminder to check Table 5 .

the familiar trigonometric tables: brackets on the left are equal to the linear combinations of those at the top, with the listed coefficients; whereas brackets on the right use these same coefficients with those at the bottom. That this arrangement, in which adjoint brackets stand at opposite ends of rows and columns, is possible is due to Corollary (V). For example, for $s=1, m=4$ we have

$$
\begin{aligned}
& {[31 e]_{23}=[40 e]_{31}+3[04 e]_{31}} \\
& {[13 e]_{31}=3[40 e]_{23}+[04 e]_{23} .}
\end{aligned}
$$

'The two equations are adjoint with respect to $k_{3}$.

\section{THE NUMBER OF NON-VANISHING IN- DEPENDENT BRACKETS}

By means of suitable counting methods, it can be shown that the number of non-vanishing independent brackets depends on $n$, the total power of $B$, as $a n^{2}+b n+c$. The values of $a, b$, and $c$ are given in Table 8 for the various symmetries.

\section{DISCUSSION}

The anisotropy of galvanomagnetic effects, first studied by RigHr in 1883, have never been analyzed comprehensively for the 32 point groups, as attempted in the present study. The resulting tables of brackets include as special cases the work of VOIGT, of JuRETSCHKE for $D_{3 i}$ and $m \leqslant 4$, of Seitz, Pearson and Suhl, and Goldberg and Davis for cubic crystals, and some of the work of KOHLER insofar as it is concordant with ONSAGER's relations. In order to facilitate the comparison of our bracket notations with the notations of some other authors, Table 9 has been prepared for the case $O_{h}$.

The experimenter can make use of the results obtained in this paper in various ways. That the Hall effect may contain odd and even terms, as reported by GoldBerg and Davis as well as by Logan and Marcus, ${ }^{(20)}$ is in perfect order. However, if magnetoresistance measurements are not free from odd terms, (17) this must be interpreted as an indication that insufficient attention has been paid to isothermal conditions in the experimental arrangement.

The tables are useful in planning and interpreting galvanomagnetic measurements, and in estimating errors due to misalignment. For these purposes the following steps are required.

According to equation (3), the measured quantity is $\rho^{\alpha 1}=F^{\alpha} / J^{1}$. In terms of the brackets one has 
L. P. KAO and E. KATZ

Table 7. Relations for non-vanishing brackets for $C_{3 i}, D_{3 i}, C_{6 h}, D_{6 h}$

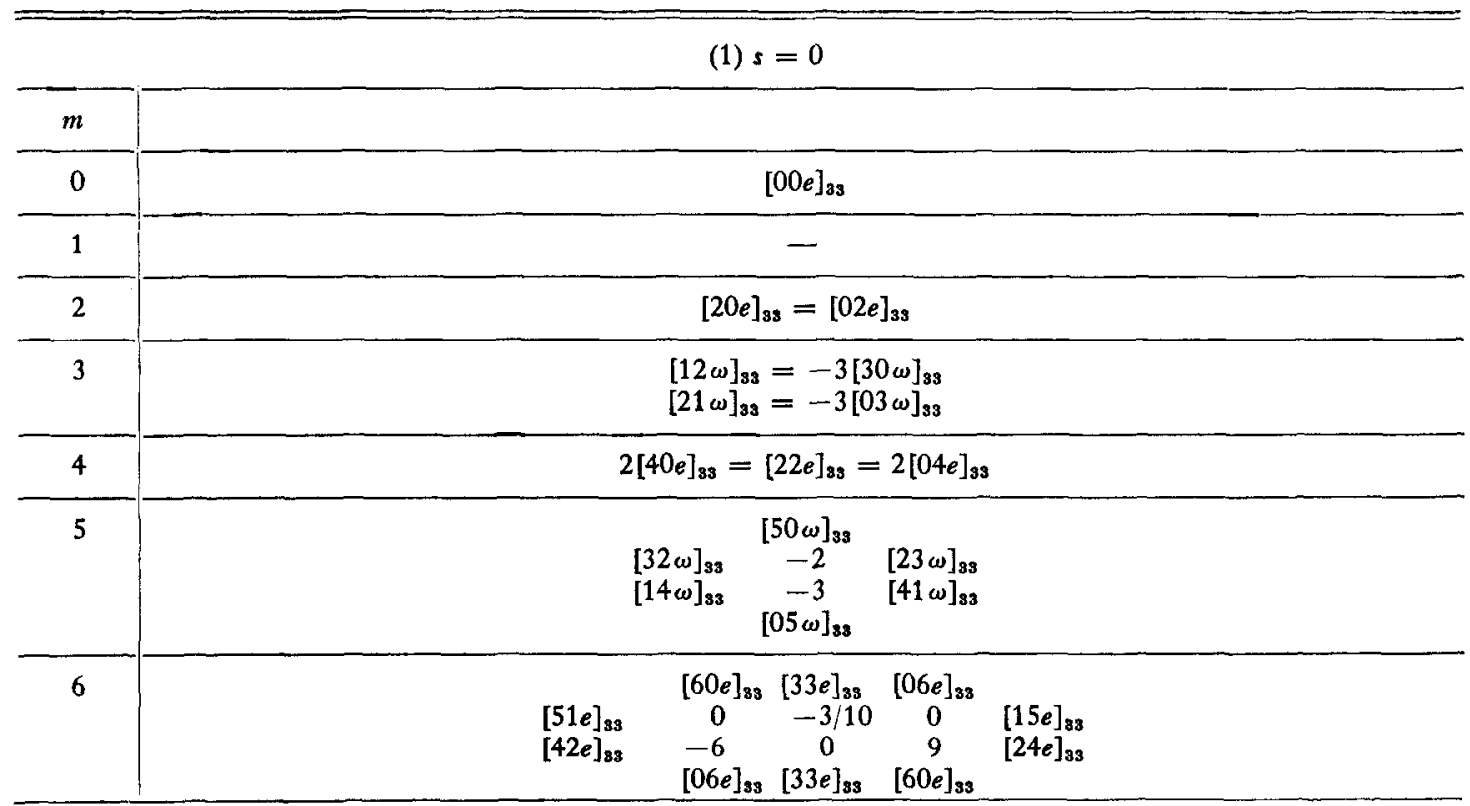

(2) $s=1 \quad n=$ even $^{*}$

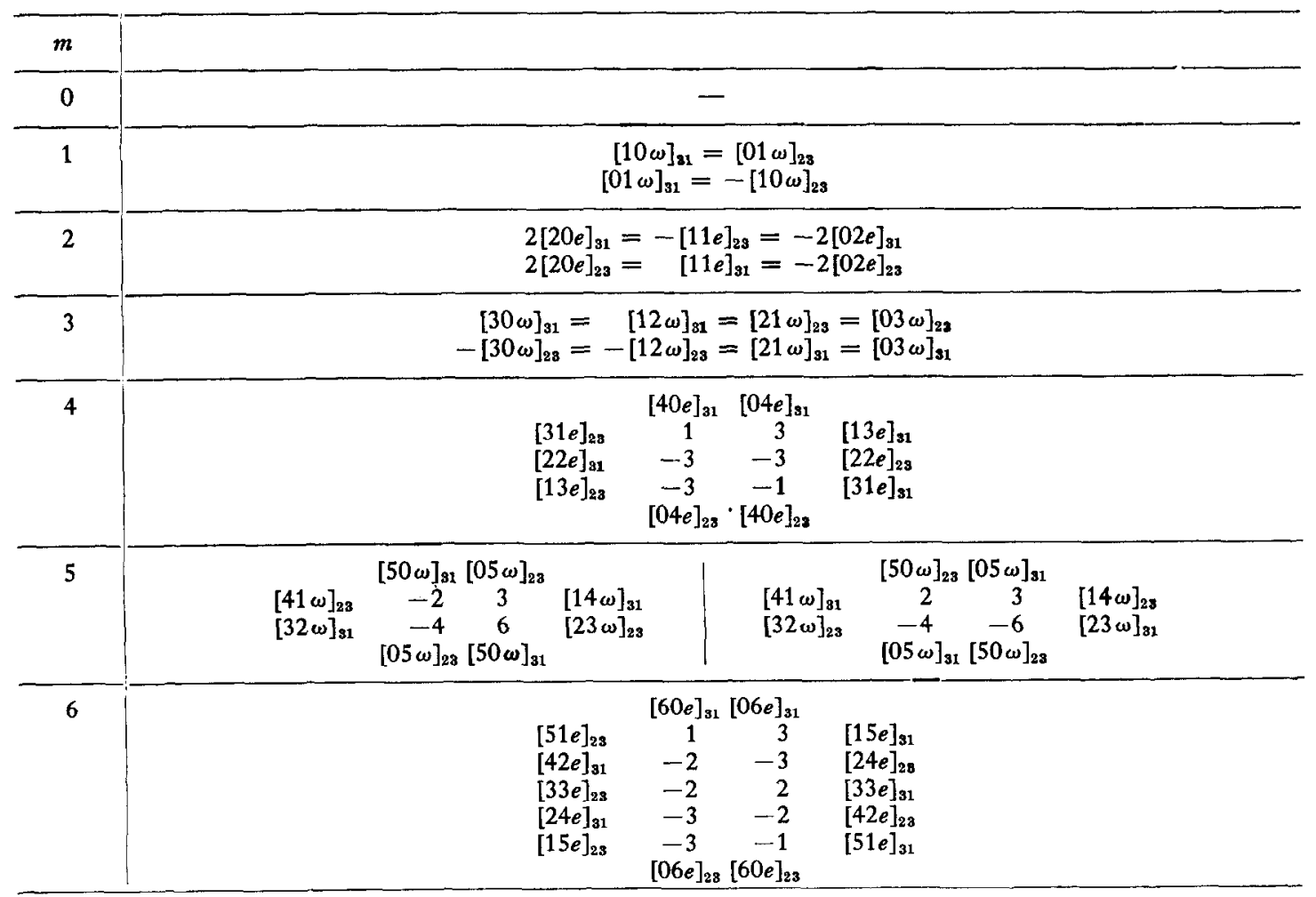

* The same table can be used for $n=$ odd if each bracket with outer indices 23 receives a minus sign and $e, \omega$ are replaced by $\omega, e$ according to Corollary (VIII). 
(3) $s=2 \quad n=$ even

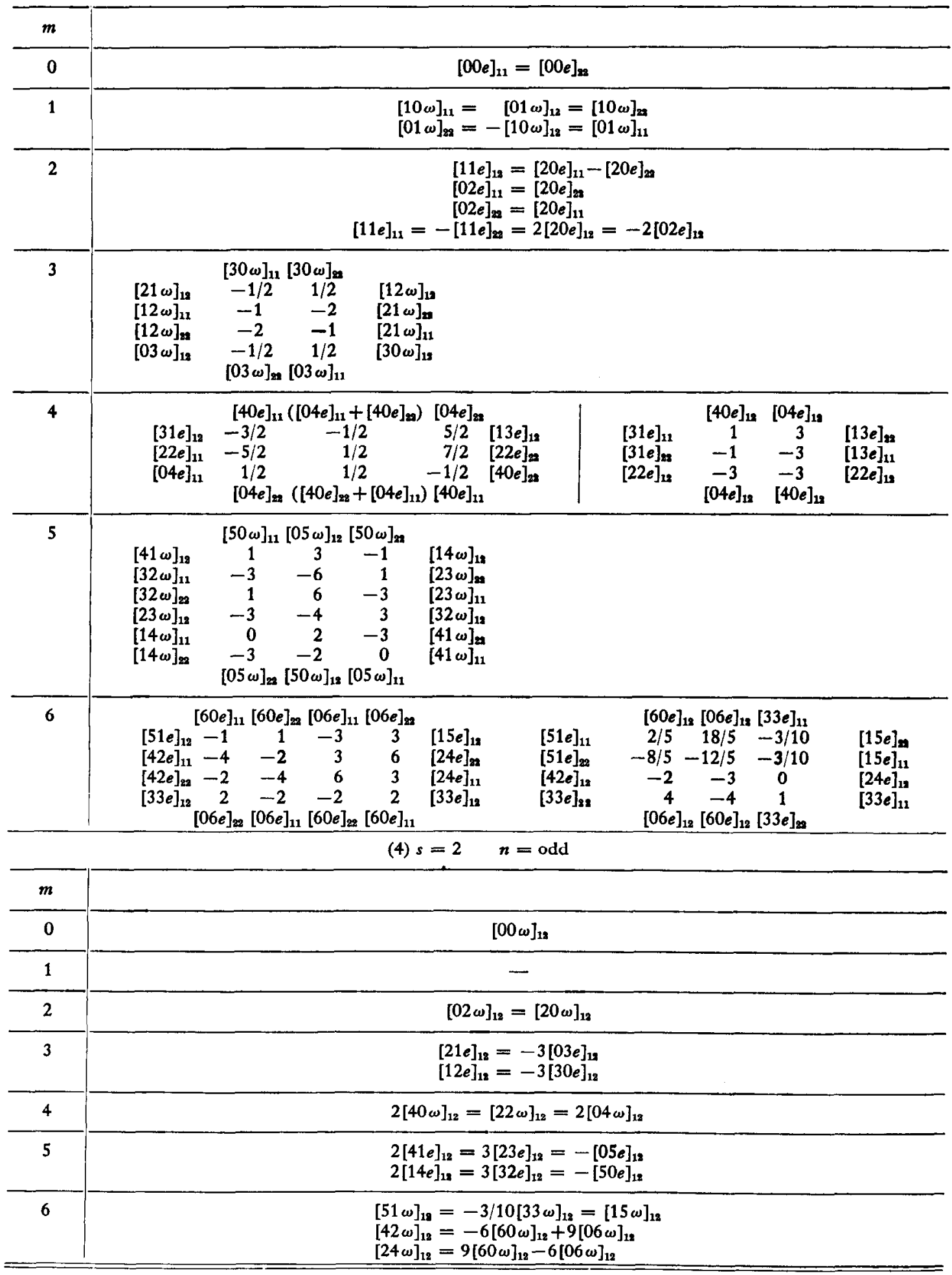


Table 8. The number of non-vanishing independent brackets $a n^{2}+b n+c$

\begin{tabular}{|c|c|c|c|c|}
\hline Group & $n$ & $a$ & $b$ & $c$ \\
\hline$C_{1}=S_{2}$ & $\begin{array}{l}\text { even } \\
\text { odd }\end{array}$ & $\begin{array}{c}3 \\
3 / 2\end{array}$ & $\begin{array}{c}9 \\
9 / 2\end{array}$ & $\begin{array}{l}6 \\
3\end{array}$ \\
\hline$C_{2 n}$ & $\begin{array}{l}\text { even } \\
\text { odd }\end{array}$ & $\begin{array}{l}3 / 2 \\
3 / 4\end{array}$ & $\begin{array}{c}5 \\
5 / 2\end{array}$ & $\begin{array}{c}4 \\
7 / 4\end{array}$ \\
\hline$C_{3 i}$ & $\begin{array}{l}\text { cven } \\
\text { odd }\end{array}$ & $\begin{array}{c}1 \\
1 / 2\end{array}$ & $\begin{array}{c}3 \\
3 / 2\end{array}$ & $\begin{array}{l}2 \\
1\end{array}$ \\
\hline$C_{4 h}$ & $\begin{array}{l}\text { even } \\
4 k+1 \\
4 k-1\end{array}$ & $\begin{array}{l}3 / 4 \\
3 / 8 \\
3 / 8\end{array}$ & $\begin{array}{l}5 / 2 \\
5 / 4 \\
5 / 4\end{array}$ & $\begin{array}{c}2 \\
11 / 8 \\
7 / 8\end{array}$ \\
\hline$C_{6 h}$ & $\begin{array}{l}6 k-2 \\
6 k \\
6 k+2 \\
6 k-1 \\
6 k+1 \\
6 k+3\end{array}$ & $\left\{\begin{array}{l}1 / 2 \\
1 / 4\end{array}\right.$ & $\left\{\begin{array}{l}5 / 3 \\
5 / 6\end{array}\right.$ & $\begin{array}{c}4 / 3 \\
2 \\
8 / 3 \\
7 / 12 \\
23 / 12 \\
5 / 4\end{array}$ \\
\hline$D_{2 h}$ & $\begin{array}{l}\text { even } \\
\text { odd }\end{array}$ & $\begin{array}{l}3 / 4 \\
3 / 8\end{array}$ & $\begin{array}{c}3 \\
3 / 2\end{array}$ & $\begin{array}{c}3 \\
9 / 8\end{array}$ \\
\hline$D_{3 i}$ & $\begin{array}{l}\text { even } \\
\text { odd }\end{array}$ & $\begin{array}{l}1 / 2 \\
1 / 4\end{array}$ & $\begin{array}{l}2 \\
1\end{array}$ & $\begin{array}{c}2 \\
3 / 4\end{array}$ \\
\hline$D_{4 h}$ & $\begin{array}{l}\text { even } \\
4 k+1 \\
4 k-1\end{array}$ & $\begin{array}{l}3 / 8 \\
3 / 16 \\
3 / 16\end{array}$ & $\begin{array}{l}7 / 4 \\
7 / 8 \\
7 / 8\end{array}$ & $\begin{array}{c}2 \\
15 / 16 \\
11 / 16\end{array}$ \\
\hline$D_{6 h}$ & $\begin{array}{l}6 k-2 \\
6 k \\
6 k+2 \\
6 k-1 \\
6 k+1 \\
6 k+3\end{array}$ & $\begin{array}{l}1 / 4 \\
1 / 8\end{array}$ & $\left\{\begin{array}{l}4 / 3 \\
2 / 3\end{array}\right.$ & $\begin{array}{c}5 / 3 \\
2 \\
7 / 3 \\
13 / 24 \\
29 / 24 \\
7 / 8\end{array}$ \\
\hline$T_{h}$ & $\begin{array}{l}\text { even } \\
\text { odd }\end{array}$ & $\begin{array}{l}1 / 4 \\
1 / 8\end{array}$ & $\begin{array}{c}1 \\
1 / 2\end{array}$ & $\begin{array}{c}1 \\
3 / 8\end{array}$ \\
\hline$O_{h}$ & $\begin{array}{l}\text { even } \\
4 k+1 \\
4 k-1\end{array}$ & $\begin{array}{l}1 / 8 \\
1 / 16 \\
1 / 16\end{array}$ & $\begin{array}{l}3 / 4 \\
3 / 8 \\
3 / 8\end{array}$ & $\begin{array}{c}1 \\
9 / 16 \\
5 / 16\end{array}$ \\
\hline Isotropic & $\begin{array}{c}\text { even } \neq 0 \\
0 \\
\text { odd }\end{array}$ & $\begin{array}{l}0 \\
0 \\
0\end{array}$ & $\begin{array}{l}0 \\
0 \\
0\end{array}$ & $\begin{array}{l}2 \\
1 \\
1\end{array}$ \\
\hline
\end{tabular}

(with the summation convention on repeated indices):

$$
\rho^{\alpha 1}(B)=\rho_{j i}(B) l_{j}^{\alpha} l_{i}^{1}=\frac{\Delta_{i j}(B)}{\Delta(B)} l_{j}^{\alpha} l_{i}^{1}
$$

Table 9. Notations of various authors for $\mathrm{O}_{h}$

\begin{tabular}{l|l}
\multicolumn{1}{c}{$\begin{array}{c}\text { SEITZ's } \\
\text { notation }\end{array}$} & \multicolumn{1}{c}{ Bracket notation } \\
\cline { 1 - 2 }$\sigma_{0}=1 / \rho_{0}$ & {$[000]_{11}$} \\
$\alpha$ & {$[100]_{23}=[001]_{12}$} \\
$\beta$ & {$[200]_{22}=[002]_{11}$} \\
$\gamma$ & {$[011]_{23}=[110]_{12}$} \\
$\beta+\gamma+\delta$ & {$[200]_{11}=[002]_{33}$} \\
& \\
$\begin{array}{c}\text { PEARSON and } \\
\text { SuHL's } \\
\text { notation } \\
a\end{array}$ & \\
$b$ & {$[100]_{23} /[000]_{11}$} \\
$c$ & $\left([200]_{22}[000]_{11}+[100]_{23}{ }^{2}\right) /[000]_{11}{ }^{2}$ \\
$d$ & $\left([100]_{23}{ }^{2}-[011]_{23}[000]_{11}\right) /[000]_{11}{ }^{2}$ \\
& $\left([200]_{11}-[200]_{22}-[011]_{23}\right) /[000]_{11}$ \\
\hline
\end{tabular}

where $\Delta_{i j}(B)$ is the cofactor of $\sigma_{i j}(B)$ in $\Delta(B)=$ det $\sigma_{i j}(B)$, and $l_{j}^{\alpha}$ is the direction cosine of the laboratory co-ordinate axis $\alpha$ with respect to the symmetry co-ordinate axis $j$. For $\alpha=1$ the equation represents the magnetoresistance and for $\alpha \neq 1$ the Hall effect. Substitution of equation (7a) into (12) leads to expressions of the form

$$
\begin{gathered}
\rho^{\alpha 1}(B)=\frac{1}{\Delta(0)}\left[\Delta(0)_{i j}^{\alpha 1} l_{j}^{\alpha} l_{i}{ }^{1}+\right. \\
\left.+Q_{i j k}^{\alpha 1} l_{j}^{\alpha} l_{i}^{1}{ }_{\gamma_{k}} B+R_{i j k l}^{\alpha 1} l_{j}^{\alpha} l_{i}{ }^{1} \gamma_{k} \gamma_{l} B^{2}+\ldots\right]
\end{gathered}
$$

where the constants $\Delta(0)_{i j}^{\alpha 1}, Q_{i j k}^{\alpha 1}, R_{i j k l}^{\alpha 1}$ can be tabulated in terms of the brackets for each crystal class. Such tables have been prepared in the thesis of Dr. KAO, but are omitted here for the sake of brevity. They are helpful in answering such questions as:

(a) How should the samples be grown $(l)$ ?

(b) How should they be placed with respect to $B(\gamma)$ for the measurement of certain brackets?

(c) What errors are caused by slight deviations from the desired $l$ or $\gamma$ values?

(d) What is the minimum number of samples, required for the determination of all brackets up to order $n$ for the various classes?

It can be shown, for example, that magnetoresistance measurements on no matter how many samples will not break up certain bracket combinations. To obtain all brackets individually, a com- 
bination of Hall and magnetoresistance measurements is best.

For the zeroth order brackets, the minimum number of samples is three for $S_{2}, C_{2 h}, D_{2 h}$, one for $T_{h}$ and $O_{h}$, and two for all others. For the firstorder brackets, the minimum number of samples is one for all classes provided the zero-order brackets are known and that Hall probes can be attached to the sample in various directions.

Finally, if among the measured brackets of order $n$ certain ones are much smaller than the majority, the tables afford an easy check whether such smallness can be interpreted as the result of a slight distortion of another lattice for which the bracket would vanish. For example, bismuth has the (augmented) group $D_{3 i}$ but can be considered as a slightly distorted case of $D_{6 h}$. Since the brackets
We prefer a pair of co-ordinate systems of a different type (complex, non-orthogonal) defined by

$$
\left.\begin{array}{l}
\overline{k_{1}{ }^{\prime}}=k_{1}{ }^{\prime}+i k_{2}{ }^{\prime} \\
\overline{k_{2}{ }^{\prime}}=k_{1}{ }^{\prime}-i k_{2}{ }^{\prime} \\
\overline{k_{3}{ }^{\prime}}=k_{3}{ }^{\prime}
\end{array}\right\}
$$

and likewise for double primes. Quantities referring to the complex co-ordinates will be marked by a bar throughout. We define the complex components of $B$ by

$$
\begin{aligned}
& \overline{B_{1}{ }^{\prime}}=B_{1}{ }^{\prime}+i B_{2}{ }^{\prime} \\
& \overline{B_{2}{ }^{\prime}}=B_{1}{ }^{\prime}-i B_{2}{ }^{\prime} \\
& \overline{B_{3}{ }^{\prime}}-B_{3}{ }^{\prime}
\end{aligned}
$$

and likewise for double primes. The complex components of the second-rank conductivity tensor are defined by*

$$
\overline{\sigma_{i j}}=\left(\begin{array}{lll}
\sigma_{11}-\sigma_{22}+i\left(\sigma_{12}+\sigma_{21}\right) & \sigma_{11}+\sigma_{22}-i\left(\sigma_{12}-\sigma_{21}\right) & \sigma_{13}+i \sigma_{23} \\
\sigma_{11}+\sigma_{22}+i\left(\sigma_{12}-\sigma_{21}\right) & \sigma_{11}-\sigma_{22}-i\left(\sigma_{12}+\sigma_{21}\right) & \sigma_{13}-i \sigma_{23} \\
\sigma_{31}+i \sigma_{32} & \sigma_{31}-i \sigma_{32} & \sigma_{33}
\end{array}\right)
$$

$[200]_{23}$ and $[011]_{11}$ vanish for $D_{6 h}$, their relative smallness for bismuth is thus made plausible.

The method of analysis developed here is applicable to a much wider field than only the galvanomagnetic effects, and could be useful whenever power series expansions with tensor coefficients of increasing rank are studied.

\section{APPENDIX}

\section{Proof of the Theorem concerning an N-Fold Rotation axis along $k_{3}$}

Let $k_{1}{ }^{\prime} k_{2}{ }^{\prime} k_{3}{ }^{\prime}$ be a set of symmetry co-ordinates of a point and let $k_{1}{ }^{\prime \prime} k_{2}{ }^{\prime \prime} k_{3}{ }^{\prime \prime}$ be the transformed set of the same point after rotation of the co-ordinate axes through an angle $\phi=2 \pi / N$ about $k_{3}$. With respect to these two systems of axes the components of a tensor $T$ of arbitrary rank are related by

$$
T^{\prime \prime}{ }_{i j \ldots l}=\sum_{t u \ldots} a_{i l} a_{j u} \ldots a_{l v} T^{\prime}{ }_{\imath u} \ldots v,
$$

where

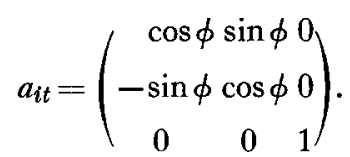

This is true in particular for the Cartesian components of the position vector $k$ of a point, of the magnetic field vector $B$, and of the components $\sigma_{i j}$ of the second-rank conductivity tensor.
According to Onsager's relation

$$
\overline{\sigma_{i j}}(B)=\overline{\sigma_{j i}}(-B),
$$

hence terms below the diagonal of equation (A.5) are dependent, and it suffices to consider those on and above the diagonal.

For convenience we shall now introduce another notation. Let $s$ denote the number of ones and twos together, and $z$ the number of twos, among the indices of $\mathrm{a} \overrightarrow{\sigma_{i j}}$. This definition of $z$ is consistent with that given

* For a tensor of arbitrary rank $n$ the complex component $\bar{T}_{i j} \ldots$ is defined as follows.

(a) Replace every index in the given order by a symbol according to this scheme: the index one by $\left(1_{1}+i_{2}\right)$, the index two by $\left(1_{1}-i_{2}\right)$, the index three by $\left(1_{3}\right)$.

(b) Multiply the $n$-fold product of symbols so obtained according to the associative and distributive law, but do not use the commutative law.

(c) Replace each "term" of the symbolic polynomial so obtained by $T$ with the indices of that term in the given order and with a coefficient equal to the product of the coefficient parts (upper parts) of the "factors" of that term. The resulting polynomial in $T$ is the desired expression.

For example, one wants to find the appropriate definition of $\bar{T}_{123}$. According to $(a)$ he writes the symbol $\left(1_{1}+i_{2}\right)\left(1_{1}-i_{2}\right)\left(1_{3}\right)$. According to $(b)$ he obtains the symbolic polynomial $\left(1_{1}\right)\left(1_{1}\right)\left(1_{3}\right)-\left(1_{1}\right)\left(i_{2}\right)\left(1_{3}\right)+\left(i_{2}\right)\left(1_{1}\right)\left(1_{8}\right)$ $-\left(i_{2}\right)\left(i_{2}\right)\left(1_{3}\right)$. According to $(c)$ the definition is now $\bar{T}_{123}=T_{113}-i T_{123}+i T_{213}+T_{223}$. 
in Section $3 b$. The numbers $s, z$ define uniquely one of the six independent pairs of indices $i j$ and vice versa. We write (note the indices between parentheses):

$$
\overline{\sigma_{(s, z)}}=\overline{\sigma_{i j}}
$$

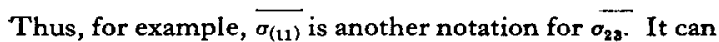
be verified by direct substitution of these definitions and comparison with equations (A.1) and (A.2) that the complex tensor components as defined transform under a rotation of co-ordinates about $k_{3}$ according to

$$
{\overline{T^{\prime \prime}}}_{i j \ldots l}=\sum_{t u \ldots v} \overline{a_{i t} a_{j u}} \ldots \overline{a_{l v}} \bar{T}_{t u \ldots v}^{\prime}
$$

with the diagonalized matrix

$$
\overline{a_{i t}}=\left(\begin{array}{lll}
e^{-i \phi} & 0 & 0 \\
0 & e^{i \phi} & 0 \\
0 & 0 & 1
\end{array}\right)
$$

In particular,

$$
\begin{aligned}
\overline{B_{1}{ }^{\prime \prime}} & =\overline{B_{1}} e^{-i \phi} \\
\overline{B_{2}{ }^{\prime \prime}} & =\overline{B_{2}} e^{i \phi} \\
\overline{B_{3}{ }^{\prime \prime}} & =\overline{B_{3}} \\
\overline{\sigma^{\prime \prime}} & \\
(8, z) & (B)=\overline{\sigma^{\prime}}(8, z)(B) e^{-i \phi(s-2 z)} .
\end{aligned}
$$

Let us write, in analogy with equation (7):

$$
\begin{aligned}
& {\overline{\sigma^{\prime}}}_{(s, z)}(B)=\sum_{n=0}^{\infty} \sum_{m=0}^{n} \sum_{w=0}^{m}{\overline{C^{\prime}}}_{(s, z)}(n, m, w) \times \\
& \times \overline{B_{1}{ }^{\prime}} w \overline{B_{2}{ }^{\prime}} m-w \overline{B_{3}{ }^{\prime} n-m} \text {. }
\end{aligned}
$$

Comparison of (A.11) with equations (7) and (8) yields

$$
\begin{gathered}
{\overline{C^{\prime}}}_{(\delta, z)}(n, m, w) \equiv(1 / 2)^{m}(-)^{w} \sum_{p=0}^{m} \sum_{\theta} g(m, p, w) \times \\
\times \in(s, \theta, z)[m-p, p, n-m]_{(s, \theta),}
\end{gathered}
$$

where $g$ and $\varepsilon$ are the functions defined in the text by equation (11) and Table 1.

If the rotation is a "covering" operation of the crystal, then the equations (A.11) and (A.12) must be invariant, i.e. their form in terms of doubly primed quantities must be identical to that in singly primed quantities, using identical brackets. The brackets are therefore written without any primes. Consequently, also

$$
{\overline{C^{\prime \prime}}}_{(s, z)}(n, m, w)=\bar{C}_{(s, z)}(n, m, w)
$$

in other words: also the primes on $\bar{C}$ can be omitted for a covering operation.
The invariance requirement applied to equation (A.11) will yield equations (9) and (10). To see this, we express the doubly primed components of $\overline{\sigma^{\prime \prime}(8, z) \text { and }}$ $\widetilde{B_{k}{ }^{\prime \prime}}$ in the doubly primed analog of (A.11) in terms of the singly primed ones by means of (A.10):

$$
\begin{aligned}
{\overline{\sigma^{\prime}}}_{(s, z)} & =\sum_{n=0}^{\infty} \sum_{m=0}^{n} \sum_{w=0}^{m} \bar{C}_{(s, z)}(n, m, w) \times \\
& \times e^{i \phi n} \overline{B_{1}{ }^{\prime}} w{\overline{B_{2}{ }^{\prime}}}^{m}-w{\overline{B_{3}}}^{\prime}{ }^{n-m}
\end{aligned}
$$

with $h=m+s-2(w+z)$. Comparing (A.14) with (A.11), it is seen in view of (A.13) that the two results are compatible only if

$$
\left.\begin{array}{lc}
\text { either } & \bar{C}_{(s, z)}(n, m, w)=0 \\
\text { or } & e^{i \phi \hbar}=1 .
\end{array}\right\}
$$

In connection with (A.12), the equations (A.15) are identical with equations (9) and (10) in the text, and the theorem is proved.

\section{REFERENCES}

1. Campbell L. L. Galvanomagnetic and Thermomagnetic Effects. Longmans, Green and Co., Inc., New York (1923).

2. MEISSNER W. Handbuch der Experimentalphysik Vol. XI, Part 2. Akademische Verlagsges., Leipzig (1935).

3. See, for example:

Logan J. K. and Marcus J. A. Phys. Rev. 88, 1234 (1952).

Willardson R. K., Harman T. C. and Beer A. C. Phys. Rev. 96, 1512 (1954).

Bullis W. M. and Krag W. E. Phys. Rev. 101, 580 (1956).

4. Casimir H. B. G. Rev. Mod. Phys. 17, 343 (1945).

Casimir H. B. G. and Gerritzen A. N. Physica 8, 1107 (1941).

5. Kapitza P. Proc. Roy. Soc. A123, 292 (1929).

6. Schubnikow L. and de HaAs W. J. Commun. Phys. Lab. Univ. Leiden No. 207a, c, d (1930); No. 210a, b (1930).

7. Stierstadt O. Z. Phys. 80, 636 (1933); 85, 310, 697 (1933); 87, 687 (1933-34); 95, 355 (1935).

8. JuSTr E. Leitfähigkeit und Leitungsmechanismus fester Stoffe, Chap. I. Vandenhoeck and Ruprecht, Göttingen (1948).

9. BLoM J. W. Magnetoresistance for Crystals of Gallium. Martinus Nijhoff, The Hague (1950).

10. Schulze R. Phys. Z. 42, 297 (1941).

TANabe Y. Sci. Rep. Res. Insts. Tôhoku Univ. 1, 275 (1949).

Gruneisen E. and Adenstedt H. Ann. Phys. 31, 714 (1937).

Lazarev B. G., Nakhimovich N. M., and ParFENova E. A. Dokl. Akad. Nauk. S.S.S.R. 24, 855 (1939). 
11. For recent works see, for example:

Pearson G. L. and Suhl. H. Phys. Rev. 83, 768 (1951).

Pearson G. L. and Herring C. Physica 20, 975 (1954).

12. VorGT W. Lehrbuch der Kristallphysik. Teubner, Leipzig and Berlin (1928).

13. Kohler M. Ann. Phys. 20, 878, 891 (1934); 95, 365 (1935).

14. Seitz F. Phys. Rev. 79, 372 (1950).

15. Juretschke H. J. Acta Cryst. 8, 716 (1955).

16. Kao L. P. and Katz E. Phys. Rev. 98, 1534 (1955).

17. References 11,4 :

Shoznderg D. Proc. Camb. Phil. Soc. 31, 271 (1935).

Meixner J. Ann. Phys. 36, 105 (1939); 40, 105 (1941).
Donovan B. and Conn G. K. T. Phil. Mag. 41, 770 (1950).

18. Goldberg C. and Davis R. E. Phys. Rev. 94, 1121 (1955).

19. WrLson A. H. The Theory of Metals p. 318. University Press, Cambridge (1953).

20. See, for example:

Alers P. B. and Webber R. T. Phys. Rev. 91, 1060 (1953).

Berlincourt T. G. and Logan J. K. Phys. Rev. 93, 348 (1954).

Berlincourt T. G. and Steele M. C. Phys. Rev. 98, 956 (1955).

Steele M. C. Phys. Rev. 99, 1751 (1955). 